\title{
pH como variável indicadora em águas subterrâneas de vazamentos provenientes de sequestro geológico de carbono
}

\author{
$p H$ as indicator variable in the groundwater of leaks from carbon geological sequestration \\ Gabriel Cavelhão ${ }^{1}$ Helen Simone Chiaranda Lazzarin ${ }^{2}$ e Henry Xavier Corseuil ${ }^{3}$
}

${ }^{1}$ Departamento de Engenharia Sanitária e Ambiental, Universidade Federal de Santa Catarina, SC, Brasil

gabriel.cavelhao@ufsc.br

${ }^{2}$ Departamento de Engenharia Sanitária e Ambiental, Universidade Federal de Santa Catarina, SC, Brasil

helen.lazzarin@ufsc.br

${ }^{3}$ Departamento de Engenharia Sanitária e Ambiental, Universidade Federal de Santa Catarina, SC, Brasil

henry.corseuil@ufsc.br

Recebido: 02/04/14 - Revisado: 30/09/14 - Aceito: 14/11/14

\begin{abstract}
RESUMO
O sequestro geológico de carbono tem se mostrado uma importante alternativa para a redução das emissões atmosféricas de $\mathrm{CO}_{2}$. $\mathrm{No}_{\text {entanto, potenciais }}$ vazamentos, provenientes da formação armazenadora, podem atingir aquiferos e comprometer sua qualidade. O desenvolvimento de técnicas para deteçãa de vazamentos pode auxiliar na agilidade de adoção de medidas mitigadoras. O uso de variáveis hidrogeoquímicas como indicadoras de exsudações pode ser uma técnica viável pelo fato de a água subterrânea poder sofrer variações expressivas em alguns parâmetros hidrogeoquímicos em fases muito iniciais de seu contato com $0 \mathrm{CO}_{2}$. Desta forma, o objetivo deste trabalho foi avaliar variáveis hidrogeoquímicas da água subterrânea que podem servir de indicadoras da interação entre $\mathrm{CO}_{2}$ e aquifero de reatividade limitada para deteç̧ão de vazamentos provenientes de sequestro geológico. Para isso, foi realizada uma simulaşão, em campo, de vaz̧amento de um sítio de armaz̧enamento geológico de $\mathrm{CO}_{2}$, por meio da injeção controlada de $\mathrm{CO}_{2}$ diretamente na porção saturada de um aquífero de reatividade limitada. O monitoramento da água subterrânea foi realizado por meio de poços multinivel e análises físico-químicas. Os resultados mostraram que as variações no $\mathrm{pH}$ podem ser indicadoras de vazamentos de $\mathrm{CO}_{2}$ provenientes de sequestro geológico em aquiferos de reatividade limitada e podem ser utilizadas em programas de Medição, Monitoramento e Verificação $(M M V)$ de $\mathrm{CO}_{2}$ sequestrado geologicamente.
\end{abstract}

Palavras Chave: Sequestro geológico de $\mathrm{CO}_{2}$. Água subterrânea. Monitoramento bidrogeoquímico. Detecção de vazamento. Aquifero de reatividade limitada

\begin{abstract}
Carbon geological sequestration has been pointed out as an important alternative for reducing atmospheric $\mathrm{CO}_{2}$ emissions. However, potential leaks from the storage formation can reach shallow freshwater aquifers and compromise their quality. The development of techniques for $\mathrm{CO}_{2}$ leak detection in shallow aquifers can assist in the prompt adoption of mitigation measures. The use of geochemical parameters as $\mathrm{CO}_{2}$ leakage detection can be a viable technique since groundwater may suffer significant variations in some geochemical parameters at very early stages of its contact with the $\mathrm{CO}_{2}$. Thus, the aim of this work was to determine hydrogeochemical variables indicative of the interaction between $\mathrm{CO}_{2}$ and a limited reactivity aquifer to detect leaks from geological sequestration. To accomplish this purpose, a field leak simulation was performed through the controlled release of $\mathrm{CO}_{2}$ in the saturated portion of a limited reactivity aquifer. Groundwater was monitored by multilevel wells and physicochemical analyses. The results showed that $p H$ variations can be indicative of leakage of $\mathrm{CO}_{2}$ from geological sequestration in limited reactivity aquifers and may be utilized in Measurement, Monitoring and Verification programs (MMV) of geologically sequestered $\mathrm{CO}_{2}$.
\end{abstract}

Keywords: $\mathrm{CO}_{2}$ geological storage. Groundwater. Geochemical monitoring. Leak detection. Limited reactivity aquifer 


\section{INTRODUÇÃO}

O sequestro geológico de carbono tem se mostrado uma tecnologia promissora para redução em curto/médio prazo das emissões de $\mathrm{CO}_{2}$, um dos principais gases que contribuem para o efeito estufa (IPCC, 2005). $\mathrm{O} \mathrm{CO}_{2}$ capturado de fontes estacionárias (indústrias, plantas energéticas) é transportado e injetado em formações geológicas profundas (campos depletados de petróleo e gás natural, jazidas de carvão e formações aquíferas salinas), que, em determinadas condições, possibilitam o armazenamento seguro de grandes volumes deste gás (BACHU; ADAMS, 2003; WHITE et al., 2003). No Brasil, um mapeamento das formações geológicas que apresentam potencial para sequestro geológico de $\mathrm{CO}_{2}$ foi realizado por meio do projeto denominado CARBMAP. Os resultados desse projeto mostraram um potencial de armazenamento de 2.035 Gton de $\mathrm{CO}_{2}$ nas bacias sedimentares brasileiras, incluindo aquíferos salinos, campos de petróleo e camadas de carvão. As bacias sedimentares do Paraná, de Santos, de Campos e do São Francisco apresentaram maior viabilidade, pela proximidade destas com as fontes estacionárias de emissões de $\mathrm{CO}_{2}$ (ROCKETT, 2011). Vazamentos do $\mathrm{CO}_{2}$ armazenado, no entanto, podem ocorrer devido à heterogeneidade do meio, presença de fraturas ou falhas geológicas e poços de extração de petróleo abandonados. Nestas condições, o $\mathrm{CO}_{2}$ tende a migrar para a superfície por ser menos denso que a água. A migração de $\mathrm{CO}_{2}$ pode atingir as águas subterrâneas rasas, alterando sua qualidade, e também se acumular em regiões próximas à superfície terrestre, trazendo riscos à população e aos seres vivos em geral (GASDA et al., 2004; KLUSMAN, 2011; NORDBOTTEN et al., 2009). Para identificar locais de vazamento de $\mathrm{CO}_{2}$, tecnologias de medição, monitoramento e verificação (MMV) têm sido estudadas. Dentre essas, destacam-se o monitoramento do fluxo de $\mathrm{CO}_{2}$ atmosférico pela Eddy Covariance (KORRE et al., 2011; LEUNING et al., 2008; LEWICKI; HILLEY, 2012) e da concentração e fluxo de gases no solo por meio de câmaras de acúmulo (JONES et al., 2009, 2011), métodos geofísicos, que determinam as variações da resistividade do meio (WHITE, 2011), monitoramento do estresse biológico, por meio de análise multiespectral de imagens da vegetação (MALE et al., 2010; ROUSE et al., 2010) e micro-organismos do solo (JONES et al., 2011; NOBLE et al., 2012), e o monitoramento hidrogeoquímico da água subterrânea por meio das alterações de variáveis indicadoras de qualidade da água (CAHILL; JAKOBSEN, 2013; FAHRNER et al., 2012; KHARAKA et al., 2010; TRAUTZ et al., 2013; WANG; JAFFE, 2004; WILKIN; DIGIULIO, 2010). Dentro desse portfólio de tecnologias disponíveis, as relacionadas à região subsuperficial têm maior potencial para detecção de $\mathrm{CO}_{2}$ em fases iniciais de vazamento, uma vez que esta é a primeira a ser impactada.

As características químicas da água, a mineralogia do aquífero e as interações resultantes destas com o $\mathrm{CO}_{2}$ determinam a magnitude das alterações hidrogeoquímicas da água subterrânea. O entendimento do comportamento do $\mathrm{CO}_{2}$ nas águas subterrâneas, com relação a sua dissolução (determinada pela variação da pressão parcial $\left(\mathrm{pCO}_{2}\right)$ ), à distribuição das espécies de carbonato e às interações com o arcabouço geológico (por meio de reações de dissolução dos minerais), são fundamentais para a determinação das variáveis hidrogeoquímicas da água subterrânea que sofrem influência devido à entrada de $\mathrm{CO}_{2}$ no aquífero. Vários estudos vêm sendo realizados com o propósito de entender tais processos, incluindo modelagem geoquímica(APPS et al., 2010; CARROLL et al., 2009; FAHRNER et al., 2012; WANG; JAFFE, 2004; WILKIN; DIGIULIO, 2010), experimentos em laboratório (CAHILL et al., 2013; LU et al., 2010; VARADHARAJAN et al., 2013) e estudos de campo (CAHILL; JAKOBSEN, 2013; KHARAKA et al., 2010; PETER et al., 2012; TRAUTZ et al., 2013). Estudos em campo, em escala piloto, vêm sendo frequentemente conduzidos pelo fato de contribuírem de maneira mais efetiva no entendimento das alterações hidrogeoquímicas da água subterrânea de cada aquífero, comparativamente a estudos de laboratório, por serem mais representativos das condições reais, que podem ser influenciadas por fatores externos (precipitação, nível do lençol freático e heterogeneidade do solo).

As características físico-químicas do solo exercem grande influência no comportamento das variáveis hidrogeoquímicas da água subterrânea na presença de $\mathrm{CO}_{2}$. Estudos de modelagem geoquímica indicam que variações no $\mathrm{pH}$, condutividade elétrica (CE), concentração de carbono inorgânico total (CIT) e metais podem indicar vazamentos de $\mathrm{CO}_{2}$, dependendo das configurações físico-químicas específicas de cada aquífero (APPS et al., 2010; CARROLL et al., 2009; FAHRNER et al., 2012; WANG \& JAFFE, 2004; WILKIN; DIGIULIO, 2010). Um dos estudos mais expressivos na área foi realizado em Montana/EUA, pelo ZERT (Zero Emission Researchand Technology Center). Nos resultados obtidos por este estudo, observou-se que a injeção controlada de $\mathrm{CO}_{2}$ em aquífero raso ( $2 \mathrm{~m}$ de profundidade) ocasionou uma elevação da acidez, alcalinidade, condutividade elétrica e concentração dos principais cátions. De acordo com os autores, os principais processos hidrogeoquímicos que influenciaram essas alterações foram a dissolução de minerais carbonatados, a dessorção e a troca de íons, dentre estes, metais tóxicos, resultantes da acidificação da água subterrânea provocada pela dissolução do $\mathrm{CO}_{2}$ injetado (KHARAKA et al., 2010). Em estudos recentes, desenvolvidos por Cahill e Jakobsen (2013), em aquífero com diferentes características físico-químicas do aquífero estudado pelo ZERT, observou-se comportamento semelhante com relação aos efeitos do $\mathrm{CO}_{2}$ na geoquímica da água subterrânea, embora, com magnitude de variação diferente. Esses estudos foram realizados em aquíferos que apresentam em sua composição minerais altamente reativos $\operatorname{com~} \mathrm{CO}_{2}$ (calcita, por exemplo).

O comportamento do $\mathrm{CO}_{2}$ em aquíferos de reatividade limitada, com predominância de quartzo e ausência de minerais carbonatados, característica de regiões de formação geológica sedimentar, ainda não foi completamente elucidado e requer estudos mais aprofundados. O objetivo deste trabalho foi avaliar variáveis hidrogeoquímicas da água subterrânea que podem servir de indicadoras da interação entre $\mathrm{CO}_{2}$ e aquífero de reatividade limitada para detecção de vazamentos provenientes de sequestro geológico. O experimento consistiu de um vazamento controlado com injeção de $\mathrm{CO}_{2}$ gasoso na subsuperfície para avaliar várias técnicas de MMV, incluindo as alterações hidrogeoquímicas na água subterrânea. Este é o primeiro laboratório de campo bra- 
sileiro realizado com o intuito de testar, implementar e validar essas técnicas.

\section{MATERIAIS E MÉTODOS}

O experimento foi conduzido na Fazenda experimental da Ressacada, pertencente à Universidade Federal de Santa Catarina em Florianópolis/SC (Fig. 1). A área experimental é de, aproximadamente, $6.280 \mathrm{~m}^{2}$ e de relevo plano. A superfície do solo é coberta por vegetação rasteira com o predomínio de gramíneas nativas do local. A condutividade hidráulica da água subterrânea situa-se entre $10^{-5}$ e $10^{-4} \mathrm{~cm} \cdot \mathrm{s}^{-1}$ (LEBAC, 2013). Determinadas em áreas próximas, a variação do nível do lençol freático foi de 0,8 a 1,8 metros e a porosidade efetiva entre 17 e $20 \%$ (RAMOS et al., 2013). Geologicamente, o local de estudo é formado por cobertura sedimentar quaternária, constituída por depósitos inconsolidados ou fracamente consolidados de areias, siltes, argilas ou conglomerados de origem marinha (IPUF, 2004).

A simulação de vazamento foi realizada por meio da injeção controlada de $\mathrm{CO}_{2}$ gasoso diretamente no aquífero (porção saturada). O poço de injeção foi projetado, construído e operado pela equipe do Laboratório de Estudos de Bacias (LEBAC) da UNESP e instalado a 3 metros de profundidade, utilizando-se a técnica direct push por meio do GeoProbe 6600. Essa técnica foi utilizada com o intuito de causar a mínima perturbação no solo, evitando, assim, a formação de caminhos preferenciais para o escape de $\mathrm{CO}_{2}$ durante a injeção. $\mathrm{O}$ poço foi revestido com tubo de PVC branco de 1" de diâmetro, em cuja extremidade inferior, foi instalada a seção filtrante para saída do $\mathrm{CO}_{2}$ (com 0,3 metro de altura). Entre 2 e 3 metros de profundidade foi adicionado um pré-filtro e entre 1 e 2 metros de profundidade, utilizou-se bentonita para selar o espaço anular. Na porção superior, o poço foi cimentado, até 1 metro de profundidade, para proteção do tubo de injeção.
A pressão de injeção de $\mathrm{CO}_{2}$ máxima admissível foi calculada para evitar a ruptura do solo e abertura de caminhos preferenciais. O cálculo foi realizado de acordo com a Equação de Payne (PAYNE et al., 2008), que leva em consideração a densidade do solo e da água e as espessuras das zonas vadosa e saturada acima do ponto de injeção. Dessa forma, a pressão máxima admissível, considerando-se um fator de segurança de $60 \%$ e as perdas de carga da tubulação, foi de até 0,27 atm. O $\mathrm{CO}_{2}$ foi injetado durante 11 dias (de 11 a 21 de setembro 2013), utilizando-se um cilindro de $\mathrm{CO}_{2}$ comprimido a alta pressão, um regulador de vazão eletroeletrônico $\left(\right.$ Serra $^{\circledR}$, modelo Smart-Trak 50 series) e um regulador de pressão analógico de duplo estágio. Durante este período foram injetados $33 \mathrm{~kg}$ de $\mathrm{CO}_{2}$ de forma contínua. A vazão mássica aplicada durante os primeiros 7 dias foi de $90 \mathrm{~g} \cdot \mathrm{h}^{-1}$ e as pressões ficaram entre 0,20 e $0,24 \mathrm{~atm}$. Nos 5 dias restantes, a vazão foi aumentada para $150 \mathrm{~g} \cdot \mathrm{h}^{-1}$ e a pressão ficou entre 0,26 e $0,34 \mathrm{~atm}$.

O perfil do solo foi caracterizado anteriormente ao período de injeção. As amostras para análise química foram coletadas até 3 metros de profundidade. Foram identificadas visualmente 3 camadas de solo de colorações distintas, as quais foram coletadas e posteriormente submetidas a análises químicas, realizadas pelo Laboratório de Solos da UDESC (Universidade do Estado de Santa Catarina). Foram determinados o pH, a quantidade de matéria orgânica, a fração de carbono orgânico, soma de bases, capacidade de troca catiônica (CTC) e volume de saturação por bases, de acordo com Tedesco et al. (1995). Análises de macro e microminerais do solo e na água subterrânea foram realizadas pelo Laboratório Natrium - Laboratórios e Engenharia Ltda. Foram determinadas as concentrações totais de alumínio (Al), antimônio (Sb), arsênio (As), bário $(\mathrm{Ba})$, berílio $(\mathrm{Be})$, boro $(\mathrm{B})$, cádmio $(\mathrm{Cd})$, cálcio $(\mathrm{Ca})$, chumbo $(\mathrm{Pb})$, cobre $(\mathrm{Cu})$, cromo $(\mathrm{Cr})$, enxofre $(\mathrm{S})$, ferro $(\mathrm{Fe})$, fósforo $(\mathrm{P})$, magnésio $(\mathrm{Mg})$, manganês $(\mathrm{Mn})$, mercúrio $(\mathrm{Hg})$, níquel $(\mathrm{Ni})$, prata $(\mathrm{Ag})$, potássio $(\mathrm{K})$, selênio $(\mathrm{Se})$, sódio $(\mathrm{Na})$, vanádio $(\mathrm{V})$ e zinco $(\mathrm{Zn})$ por meio

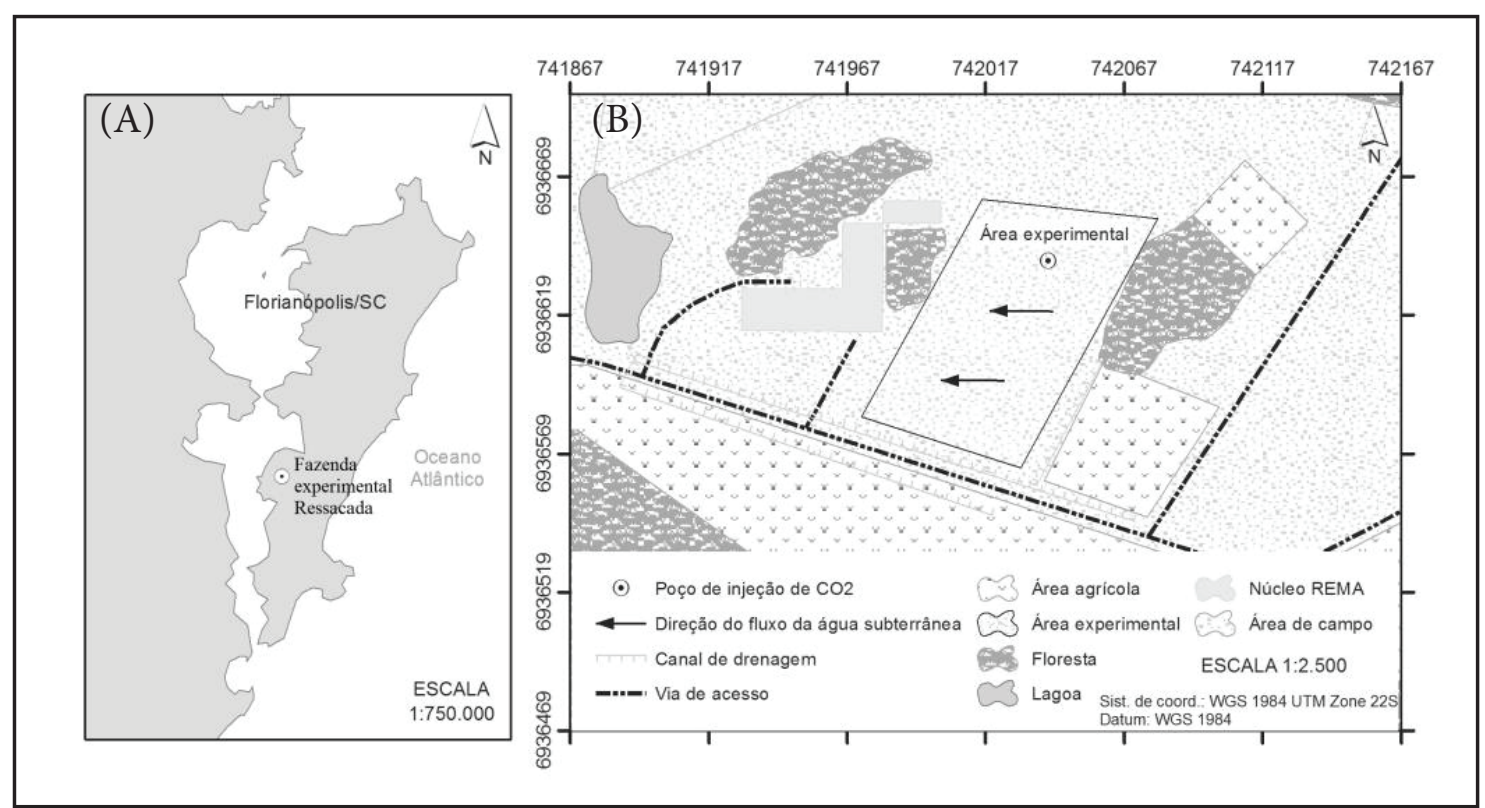

Figura 1 - Mapa de Florianópolis/SC com a localização da Fazenda experimental da Ressacada (A) e mapa da área experimental com as principais características do entorno (B) 
de espectrometria de emissão ótica acoplada a plasma indutivo (ICP-OES), de acordo com o Standard Methods - Método 3120B (APHA, 1992a), com limite de quantificação de 0,005 mg.L $\mathrm{L}^{-1}$. A coleta das amostras para análise física foi realizada no perfil do solo até 4 metros de profundidade e distante 7 metros do poço de injeção. A caracterização foi realizada pelo Laboratório de Estudos de Bacias (LEBAC) da UNESP, por meio de análise granulométrica com peneiramento e sedimentação, de acordo com a NBR 7181 (ABNT, 1984), e identificação da mineralogia pela análise de difração de raios-X, por meio de analisador portátil de XRD $\left(\right.$ Olympus $\left.^{\circledR}\right)$.

O monitoramento hidrogeoquímico da água subterrânea foi realizado com a coleta de amostras por meio de poços multinível instalados no entorno do poço de injeção e no sentido do fluxo da água subterrânea. Foram instalados 5 poços, cada um com 3 níveis de profundidade (2, 4 e $6 \mathrm{~m}$ ) (Figuras 2 e 3). Os poços foram construídos utilizando-se mangueiras de PEBD (polietileno de baixa densidade) com diâmetros de 3/16". Filtros de aço inox (com malha \#100) foram colocados nas extremidades das mangueiras. A instalação dos poços foi realizada com o auxílio de equipamento mecânico de perfuração, evitando-se a perturbação do meio e a abertura de caminhos preferenciais para escape de $\mathrm{CO}_{2}$. As amostras foram coletadas antes, durante e após o período de injeção, totalizando 23 campanhas de amostragem de água subterrânea realizadas durante um período de 40 dias (entre 2 de setembro e 10 de outubro de 2013).

As amostras de água subterrânea foram coletadas utilizando-se bomba peristáltica (Millipore ${ }^{\circledR}$, modelo Easy-Load). Para cada nível de amostragem, foi utilizada uma mangueira de polipropileno da Masterflex ${ }^{\mathbb{B}}$ Tygon, evitando-se problemas de contaminação cruzada. Análises de temperatura, pH, condutividade elétrica, potencial de oxidação-redução, salinidade e oxigênio dissolvido foram realizadas diretamente no campo, utilizando-se um analisador FlowCell, modelo MP20 (MicroPurge $\left.^{\circledR}\right)$. Amostras de $250 \mathrm{~mL}$ foram coletadas e analisadas em laboratório para determinação de alcalinidade, acidez, ferro ferroso $\left(\mathrm{Fe}^{2+}\right)$ e os ânions brometo $\left(\mathrm{Br}^{-}\right)$, cloreto $\left(\mathrm{Cl}^{-}\right)$, nitrato $\left(\mathrm{NO}_{3}{ }^{-}\right)$, nitrito $\left(\mathrm{NO}_{2}^{-}\right)$, fosfato $\left(\mathrm{PO}_{4}^{3-}\right)$, sulfato $\left(\mathrm{SO}_{4}^{2-}\right)$ e acetato $\left(\mathrm{CH}_{3} \mathrm{COO}^{-}\right)$. A determinação da acidez e da alcalinidade teve como base o método titrimétrico de acordo com o Standard Methods - Métodos 2310B (APHA, 1992b) e 2320B (APHA, 1992c). A análise de $\mathrm{Fe}^{2+}$ foi conduzida em espectrofotômetro $\mathrm{HACH}^{\circledR}$ - DR/2500, de acordo com Standard Methods - Método 8146 (APHA, 1992d). Os ânions $\mathrm{Br}^{-}, \mathrm{Cl}^{-}, \mathrm{NO}_{3}^{-}, \mathrm{NO}_{2}^{-}, \mathrm{PO}_{4}^{3-}$, $\mathrm{SO}_{4}^{2-}$ e $\mathrm{CH}_{3} \mathrm{COO}^{-}$foram analisados por cromatografia iônica, seguindo método 300.00 da USEPA (USEPA, 1993), em cromatógrafo de íons da Dionex (modelo ICS-1000), equipado com detector de condutividade iônica e coluna AS22 (4 x 250 mm). As análises de macro e microminerais na água subterrânea foram determinadas no período anterior à injeção, para determinação das condições originais da área experimental (background), e 15 dias após o término da injeção.

Os dados foram avaliados estatisticamente para verificação de diferenças entre os períodos do experimento, (background (dias -10 a 0), injeção (dias 1 a 11) e pós-injeção (dias 12 a 30)). Realizou-se comparação entre medianas por meio do teste não paramétrico de Kruskal-Wallis. O software utilizado foi o Statistica $8.0^{\circledR}$.

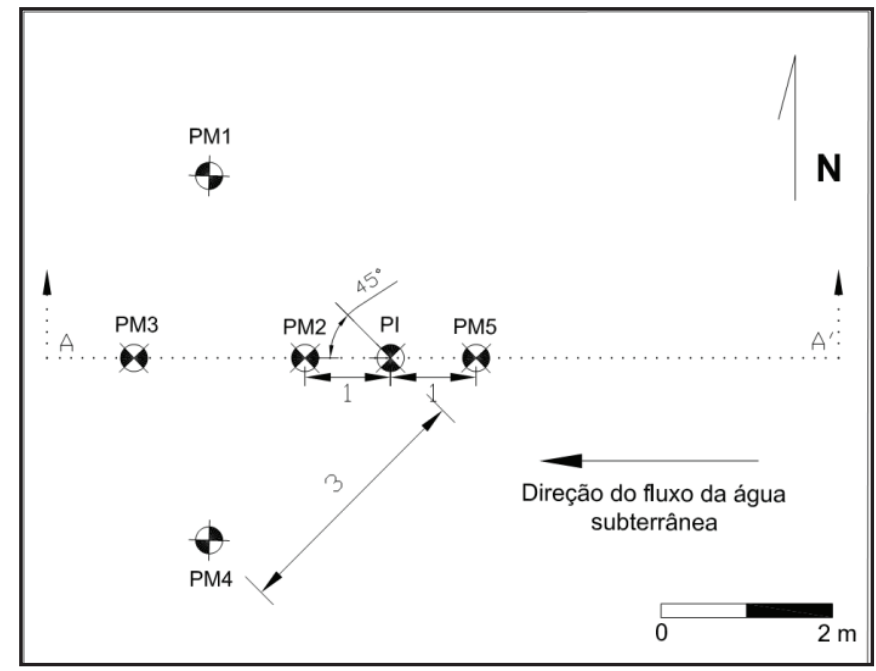

Figura 2 - Vista em planta da distribuição dos poços de monitoramento (PM) da água subterrânea na área experimental em relação ao poço de injeção (PI) (escala em metros)

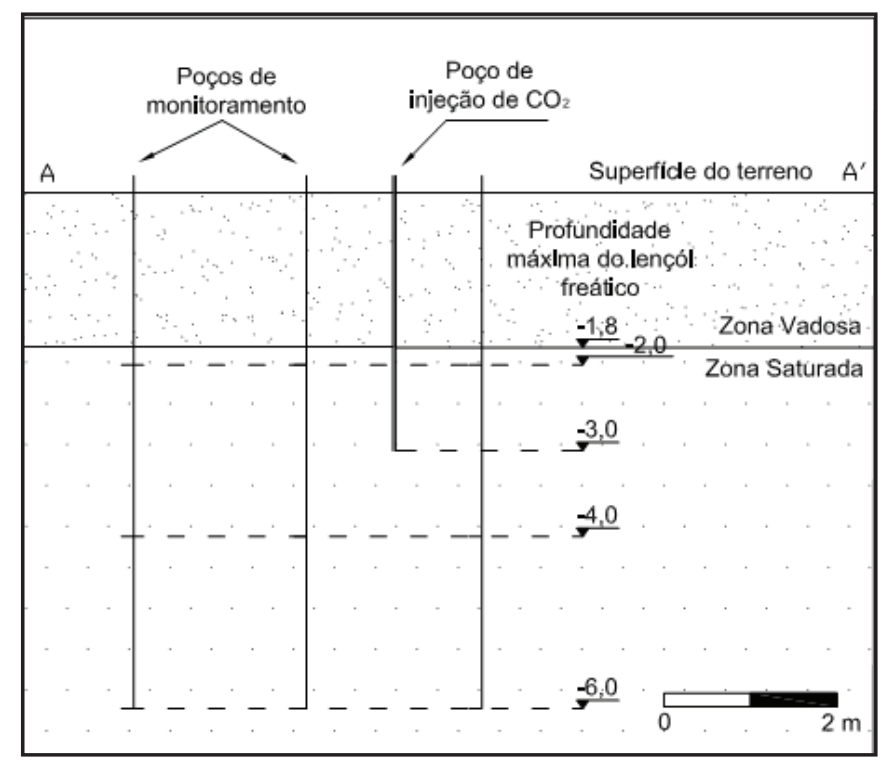

Figura 3 - Vista em corte da distribuição dos poços de monitoramento (PM) da água subterrânea na área experimental em relação ao poço de injeção (PI) (escala em metros)

\section{RESULTADOS E DISCUSSÃO}

As características químicas e mineralógicas do aquífero determinam a magnitude dos efeitos da dissolução do $\mathrm{CO}_{2}$ na água. De acordo com a análise do perfil de solo em amostras coletadas no local de instalação do poço de injeção, observouse a predominância de areias, intercaladas com finas camadas de silte e argila. Da superfície até 1 metro de profundidade, o solo apresentou maior teor de argila e matéria orgânica, resultantes, principalmente, da decomposição de restos de vegetais, pH ácido $(5,0)$ e elevada concentração de alumínio $(41 \%$ da saturação) (Tabela 1). No perfil subjacente, entre 1 e 3 metros de profundidade, o solo apresentou baixo teor de matéria orgânica $(0,3 \%)$, pH próximo a 6 (Tabela 1$)$ e houve predomínio 
Tabela 1 - Caracterização físico-química do solo

\begin{tabular}{|c|c|c|c|c|c|c|c|c|c|c|c|}
\hline \multirow[t]{2}{*}{ Perfil de coleta } & \multirow[t]{2}{*}{$\mathrm{pH}$} & \multicolumn{2}{|l|}{ CTC efetiva } & Alumínio & Bases & $\begin{array}{l}\text { Matéria } \\
\text { Orgânica }\end{array}$ & & $\begin{array}{l}\text { Carbono } \\
\text { Orgânico }\end{array}$ & Argila & \multicolumn{2}{|c|}{$\mathrm{Ag}$} \\
\hline & & \multicolumn{2}{|l|}{ cmolc. $\mathrm{dm}^{-3}$} & \multicolumn{2}{|c|}{ Saturação $(\%)$} & \multicolumn{4}{|c|}{ - } & \multicolumn{2}{|c|}{ mg.100mL-1 } \\
\hline 50 a $90 \mathrm{~cm}$ & 5,0 & \multicolumn{2}{|l|}{2,4} & 40,8 & 11,5 & \multicolumn{2}{|c|}{2,2} & 1,3 & 15,0 & \multicolumn{2}{|c|}{68,9} \\
\hline 156 a $176 \mathrm{~cm}$ & 5,7 & \multicolumn{2}{|l|}{1,2} & 0,0 & 21,4 & \multicolumn{2}{|l|}{0,3} & 0,2 & 7,0 & \multicolumn{2}{|c|}{43,9} \\
\hline 275 a $300 \mathrm{~cm}$ & 5,9 & \multicolumn{2}{|l|}{1,8} & 0,0 & 64,4 & \multicolumn{2}{|l|}{0,2} & 0,1 & 7,0 & \multicolumn{2}{|c|}{37,7} \\
\hline & $\mathrm{Al}$ & $\mathrm{Ca}$ & $\mathrm{Cu}$ & $\mathrm{S}$ & $\mathrm{Fe}$ & $\mathrm{P}$ & $\mathrm{Mg}$ & $\mathrm{Mn}$ & $\mathrm{K}$ & $\mathrm{Na}$ & $\mathrm{Zn}$ \\
\hline & \multicolumn{11}{|c|}{----mg.kg-11---- } \\
\hline 50 a $90 \mathrm{~cm}$ & 1 & 2,7 & 0,6 & 0,05 & 51,1 & 31,5 & 0,2 & 5,0 & 100,5 & 76,2 & 0,4 \\
\hline 156 a $176 \mathrm{~cm}$ & $<$ L.Q. ${ }^{1}$ & 2,9 & 0,5 & $<$ L.Q. $^{2}$ & 50,6 & 18,4 & 0,1 & 1,2 & 125,8 & 71,9 & 0,3 \\
\hline 275 a $300 \mathrm{~cm}$ & <L.Q. ${ }^{1}$ & 2,2 & 0,3 & $<$ L.Q. ${ }^{2}$ & 8,1 & 1,1 & 0,1 & 0,1 & 73,4 & 70,1 & 0,1 \\
\hline
\end{tabular}

${ }^{1} \mathrm{~L} . \mathrm{Q} .=$ Limite de quantificação $=0,005 \mathrm{mg} \cdot \mathrm{L}^{-1}{ }^{2}{ }^{2} \mathrm{~L} \cdot \mathrm{Q} .=0,05 \mathrm{mg} \cdot \mathrm{L}^{-1}$

Tabela 2 - Caracterização físico-química da água subterrânea

\begin{tabular}{|c|c|c|c|c|c|c|c|c|c|c|}
\hline & \multirow{2}{*}{\multicolumn{2}{|c|}{$\begin{array}{c}\text { Temperatura } \\
\left({ }^{\circ} \mathrm{C}\right)\end{array}$}} & \multirow{2}{*}{$\mathrm{pH}$} & Acidez $\quad A$ & Alcalinidade & \multirow{2}{*}{\multicolumn{3}{|c|}{$\begin{array}{l}\text { Condutividade } \\
\text { elétrica }\left(\mu \mathrm{S} . \mathrm{cm}^{-1}\right)\end{array}$}} & \multirow{2}{*}{$\begin{array}{c}\text { OD } \\
\left(\mathrm{mg} \cdot \mathrm{L}^{-1}\right)\end{array}$} & \multirow{2}{*}{$\begin{array}{l}\text { Potencial } \\
\text { redox }(\mathrm{mV})\end{array}$} \\
\hline & & & & \multicolumn{2}{|c|}{$\mathrm{mgCaCO}_{3} \mathrm{~L}^{-1}$} & & & & & \\
\hline Média & \multicolumn{2}{|c|}{20,61} & 5,17 & 32,63 & 2,35 & & 38,18 & & 1,95 & 285,74 \\
\hline Desvio padrão & \multicolumn{2}{|c|}{0,74} & 3,16 & 9,10 & 0,71 & & 5,49 & & 1,35 & 60,37 \\
\hline \multirow{5}{*}{$\begin{array}{c}\text { Média } \\
\text { Desvio padrão }\end{array}$} & \multirow{2}{*}{\multicolumn{2}{|c|}{$\begin{array}{c}\text { Salinidade } \\
(\% 0)\end{array}$}} & $\mathrm{Fe}^{2+}$ & $\mathrm{CH}_{3} \mathrm{COO}^{-}$ & $\mathrm{Cl}^{-}$ & & & $\mathrm{NO}_{3}^{-}$ & $\mathrm{PO}_{4}{ }^{3-}$ & $\mathrm{SO}_{4}^{2-}$ \\
\hline & & & & & & $----m g$ & $1---$ & & & \\
\hline & \multirow{2}{*}{\multicolumn{2}{|c|}{$\begin{array}{c}0,02 \\
4,50 \mathrm{E}-03 \\
\end{array}$}} & 0,15 & 0,24 & 5,27 & & & 0,30 & 0,01 & 5,29 \\
\hline & & & 0,34 & 0,31 & 0,93 & & & 0,35 & 0,02 & 1,44 \\
\hline & $\mathrm{Mg}$ & $\mathrm{Na}$ & $\mathrm{K}$ & $\mathrm{S}$ & $\mathrm{Al}$ & $\mathrm{Sb}$ & $\mathrm{Be}$ & $\mathrm{Fe}$ & $\mathrm{Ag}$ & $\mathrm{Se}$ \\
\hline & \multicolumn{10}{|c|}{----mg.L -1---- $^{-1}$} \\
\hline Média & 0,076 & 0,759 & 4,912 & 0,167 & 0,056 & 0,745 & 0,668 & 0,307 & 0,324 & 1,199 \\
\hline Desvio Padrão & 0,031 & 0,115 & 2,670 & 0,111 & - & - & 0,328 & - & 0,069 & 0,630 \\
\hline
\end{tabular}

de material arenoso, com maiores frações de areia fina à média com poucos finos $(3,8 \%$ de areia grossa, $61,8 \%$ de areia média, $30,5 \%$ de areia fina, $1,0 \%$ de silte e $2,5 \%$ de argila). A mineralogia de cada fração desse material foi identificada por meio de difração de raios-X.Na fração de areia predominaram minerais de quartzo $\left(\mathrm{SiO}_{2}\right)$ e nas frações de silte e argila predominaram argilominerais como a caulinita $\left(\mathrm{Al}_{2} \mathrm{Si}_{2} \mathrm{O}_{5}(\mathrm{OH})_{4}\right)$ e a ilita $((\mathrm{Al}, \mathrm{Mg}$, $\left.\mathrm{Fe})_{2}(\mathrm{Si})_{4} \mathrm{O}_{10}\left[(\mathrm{OH})_{2}, \mathrm{nH}_{2} \mathrm{O}\right]\right)$. O predomínio de areia quartzosa e apequena fração de argilominerais caracterizam o aquífero como sendo de reatividade limitada, com baixa concentração de íons dissolvidos. Isto ocorre devido às pequenas taxas de dissolução do quartzo (entre $10^{-14}$ e $10^{-16} \mathrm{~mol} \cdot \mathrm{m}^{-2} \cdot \mathrm{s}^{-1}$ ) e da caulinita e ilita (entre $10^{-12}$ e $10^{-13} \mathrm{~mol} \cdot \mathrm{m}^{-2} \cdot \mathrm{s}^{-1}$ )(SVERDRUP; WARFVINGE, 1988). Além disso, a água apresenta baixa alcalinidade $(<4,5$ $\left.\mathrm{mgCaCO} . \mathrm{L}^{-1}\right)$ devido à ausência de minerais carbonatados,

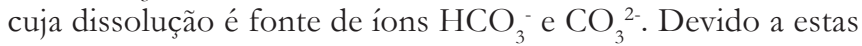
características, a água é naturalmente ácida $(\mathrm{pH} \approx 5)$ (Tabela 2) e não apresenta capacidade de tamponamento.

Com a injeção de $\mathrm{CO}_{2}$ gasoso no aquífero, verificou-se um aumento da acidificação da água subterrânea. Esse efeito foi monitorado por meio das variações de $\mathrm{pH}$ e acidez ao longo do período experimental. Foram observadas variações nesses parâmetros em todos os poços de monitoramento e não foram observadas diferenças significativas entre os níveis de amostragem (2, 4 e 6 metros). Em relação ao pH, as maiores variações foram observadas nos PMs 2 e 4, locais em que foram constatadas variações significativas $(\mathrm{p}<0,05)$ entre os períodos de background e injeção de $\mathrm{CO}_{2}$. O valor mínimo de $\mathrm{pH}$ observado no período de injeção foi de 4,1, em ambos os poços, com variação de 0,5 unidade em relação aos valores mínimos observados no background (4,6)(Figuras. 4A e 4B).

A acidificação da água subterrânea é determinada, principalmente, em função do aumento da pressão parcial de $\mathrm{CO}_{2}\left(\not \mathrm{CO}_{2}\right)(\mathrm{BUTLER}, 1982$; FETTER, 1994). A $p$ CO 2 na saída do poço de injeção variou entre 0,20 e 0,34 atm e, considerando que a solubilidade do $\mathrm{CO}_{2}$ em soluções ácidas $(\mathrm{pH}<5)$ é governada pela Lei de Henry (Eq. 1), a concentração máxima de $\mathrm{CO}_{2}$ dissolvido variou entre 0,007 e 0,011 mol.L ${ }^{-1}$. Devido à baixa concentração de íons dissolvidos na água subterrânea da área experimental, pode-se determinar a concentração de íons $\mathrm{H}^{+}$analogamente ao processo de dissolução de $\mathrm{CO}_{2}$ em águas puras (isenta de substâncias dissolvidas), negligenciando as concentrações $\mathrm{CO}_{3}{ }^{2-}$ e $\mathrm{OH}^{-}$. Assim, combinando a equação da Lei de Henry (Eq. 1) à equação de ionização do ácido carbônico (Eq. 2), obteve-se a Eq. 3, que é utilizada para estimar a concentração de íons $\mathrm{H}^{+}$(BUTLER, 1982). Sendo assim, o $\mathrm{pH}$ teórico mínimo estimado devido ao aumento da $p \mathrm{CO}_{2}$, e sua consequente dissolução, foi de 4,1 , valor observado no experimento, confirmando a acidificação do meio em função do aumento da $p \mathrm{CO}_{2}$.

$$
\left[\mathrm{CO}_{2}\right]=K_{\mathrm{H}} \cdot \mathrm{p} \mathrm{CO}_{2}
$$


$\left[\mathrm{H}^{+}\right] \cdot\left[\mathrm{HCO}_{3}^{-}\right]=K_{a 1} \cdot\left[\mathrm{H}_{2} \mathrm{CO}_{3}{ }^{*}\right]$

$\left[H^{+}\right]^{2}=K_{a 1} \cdot K_{H} \cdot p C O_{2}$

em que:

$\mathrm{H}_{2} \mathrm{CO}_{3}^{*}=\left[\mathrm{CO}_{2}(\mathrm{aq})\right]+\left[\mathrm{H}_{2} \mathrm{CO}_{3}\right](\mathrm{BUTLER}, 1982 ; \mathrm{STUMM} ;$ MORGAN, 1981)

$\mathrm{K}_{\mathrm{H}}=10^{-1,4} \mathrm{~mol} \cdot \mathrm{L}^{-1} \cdot \mathrm{atm}^{-1}$ a $20^{\circ} \mathrm{C}$ (BUTLER, 1982)

$\mathrm{K}_{\mathrm{a} 1}=10^{-6,4} \mathrm{~mol} \cdot \mathrm{L}^{-1} \cdot \mathrm{atm}^{-1}$ a $20^{\circ} \mathrm{C}(\mathrm{BUTLER}, 1982)$

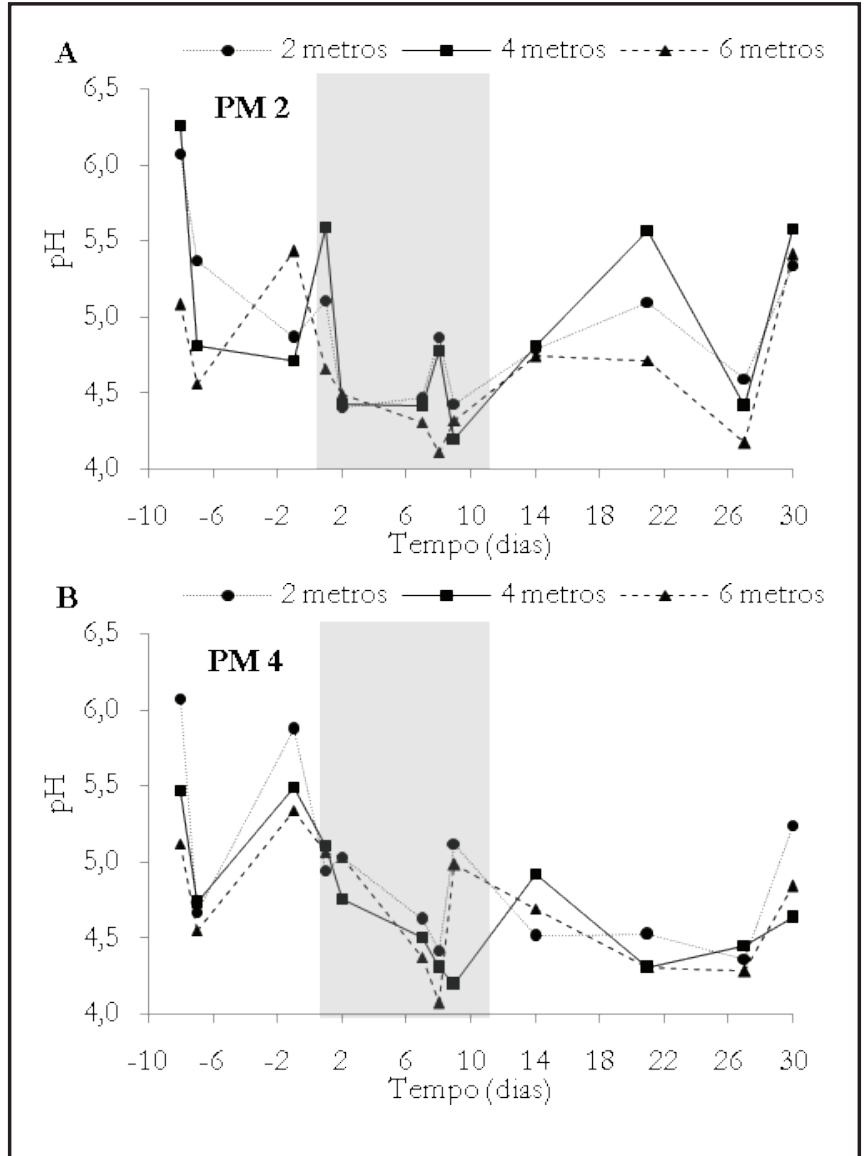

Figura 4 - Variação do pH no PM 2 (A) e no PM 4 (B) durante o experimento. A área sombreada destaca o período de injeção de $\mathrm{CO}_{2}$ na água subterrânea (de 0 a 11 dias).

Além da influência da pressão parcial de $\mathrm{CO}_{2}$ injetada, a amplitude de variação do $\mathrm{pH}$ depende da composição mineralógica do aquífero. Devido ao $\mathrm{pH}$ ser naturalmente inferior em aquíferos de reatividade limitada, sua amplitude de diminuição, devido à dissolução de $\mathrm{CO}_{2}$, é inferior. Estudos têm mostrado variações no $\mathrm{pH}$ em uma unidade (de 5,8 a 4,8) em aquíferos menos reativos (LU et al., 2012), comparados a aquíferos com maior fração de mineral reativo, em que as variações foram de 1,4 unidades (de 7,0 a 5,6) (KHARAKA et al., 2010).

A acidificação do meio também foi monitorada pelas variações na acidez. Embora estas variações não tenham apresentado diferenças significativas $(p>0,05)$ entre os períodos de background, injeção e pós-injeção, foi verificada uma tendência de aumento desta variável nos PM 2, 4 e 5, com valores máximos próximos a $115 \mathrm{mgCaCO} . \mathrm{L}^{-1}$ no PM 5 (nível 2 metros) no período de pós-injeção (Figura $5 \mathrm{~A}$ ). Não se descarta a possibilidade de ocorrência de desgaseificação das amostras coletadas em função da mudança de ambiente e consequente alteração da $p \mathrm{CO}_{2}$. A acidificação da água subterrânea também pode ter sido significativamente influenciada pelos eventos de precipitação ocorridos durante o experimento. $\mathrm{Na}$ fase final do período de injeção (dias 10, 11 e 12), ocorreram chuvas intensas (cerca de $170 \mathrm{~mm}$ acumulados em 3 dias (Figura 5B)) que causaram, inclusive, a inundação da área.

A dissolução de $\mathrm{CO}_{2}$ em aquífero de reatividade limitada mantém a alcalinidade com concentrações estáveis. Durante o experimento, a alcalinidade se manteve constante $\left(<4,5 \mathrm{mgCaCO} \cdot \mathrm{L}^{-1}\right)$. A dissolução de $\mathrm{CO}_{2}$ na água não altera a alcalinidade, em função da espécie de carbonato predominante estar na forma de $\mathrm{CO}_{2}$ dissolvido $\left(\mathrm{pK}_{\mathrm{a} 1}<6,3\right)$ (BUTLER, 1982; STUMM; MORGAN, 1981). Nessas condições, o aumento da alcalinidade, dado pelo incremento das concentrações de $\mathrm{HCO}_{3}^{-} \mathrm{e}$ $\mathrm{CO}_{3}^{2-}$, somente é possível em função da dissolução de minerais reativos (calcita, por exemplo). Em aquíferos com presença de calcita, o aumento da alcalinidade proveniente da dissolução de

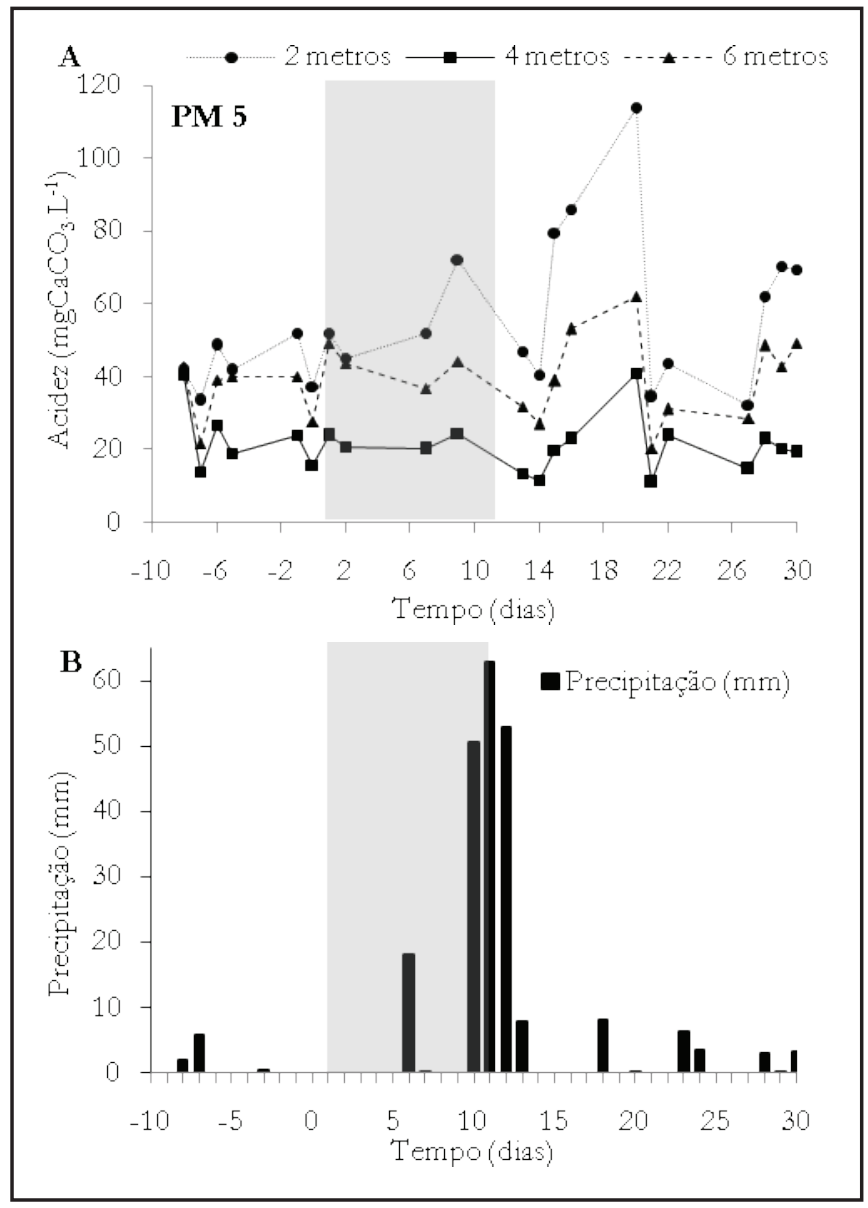

Figura 5 - Variação da acidez no PM 5 (A) e valores de precipitação ocorridos na área experimental (B) durante o experimento. A área sombreada destaca o período de injeção de $\mathrm{CO}_{2}$ na água subterrânea (de 0 a 11 dias) 


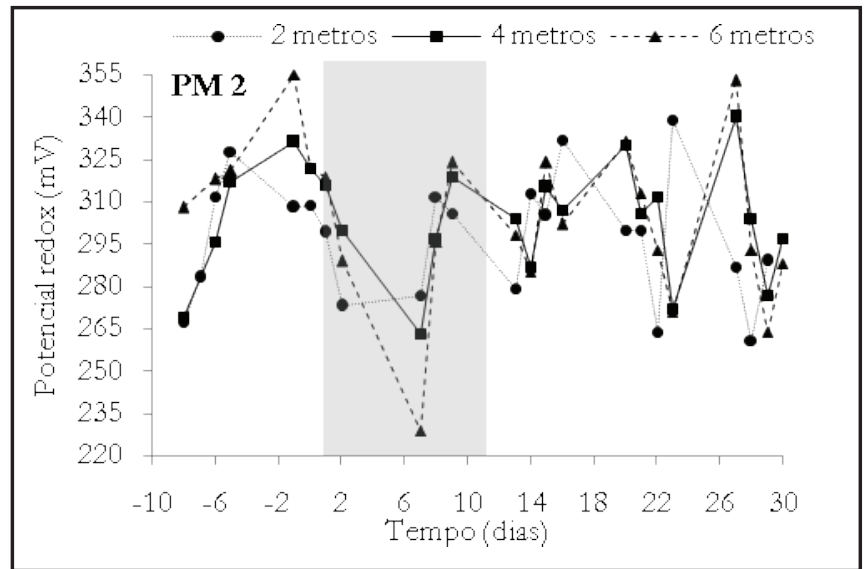

Figura 6 - Variação do potencial de oxidação-redução no PM 2 durante o experimento. A área sombreada destaca o período de injeção de $\mathrm{CO}_{2}$ na água subterrânea

$\mathrm{CaCO}_{3}$ e ionização de $\mathrm{H}_{2} \mathrm{CO}_{3}$ e $\mathrm{HCO}_{3}$, é significativa, chegando a valores próximos a $1.300 \mathrm{mg} \cdot \mathrm{L}^{-1}$, com aumento de $325 \% \mathrm{em}$ relação ao background (KHARAKA et al., 2010). No aquífero estudado, a alcalinidade não sofre alteração pela presença de $\mathrm{CO}_{2}$ e dessa forma não é indicadora de vazamentos.

Pequenas variações no potencial de oxidação-redução (Eh) foram observadas na região próxima ao poço de injeção de $\mathrm{CO}_{2}$. Observou-se uma tendência de diminuição deste potencial, principalmente no PM 2, a 6 metros de profundidade (Figura 6), com variação de 308 para $229 \mathrm{mV}$, entre os períodos de background e injeção, no entanto, essa variação não foi significativa estatisticamente $(p>0,05)$, quando comparadas as variações entre os períodos. O reestabelecimento das concentrações de background foi observado ainda no período de injeção de $\mathrm{CO}_{2}$ (a partir do $8^{\circ}$ dia), podendo ter sido influenciado pelos eventos de precipitação nesse período (Figura 5B). Em estudos de modelagem geoquímica, em aquífero reativo, também foram observadas mudanças da condição redox, devido à rápida depleção de $\mathrm{O}_{2}$ na zona de injeção de $\mathrm{CO}_{2}$ (ALTEVOGT; JAFFE, 2005). A estagnação nas reações de oxidação, também proporcionou o aumento da concentração de matéria orgânica dissolvida. Em outro estudo, as condições anóxicas proporcionadas pelo fluxo de $\mathrm{CO}_{2}$ gasoso causaram aumento da dissolução de espécies reduzidas de metais (Mn, Fe e Co) (ARDELAN, 2010).

Alterações na concentração e mobilidade de íons foram observadas na água subterrânea. $\mathrm{O}$ aumento da concentração de metais traço (B, Sb, As, Cd, Pb, Cr e Ag) excederam os valores máximos aceitáveis para qualidade da água subterrânea (CONAMA 420/2009)(Tabela 3). Esse incremento foi favorecido pelo aumento da concentração de íons $\mathrm{H}^{+}$, provenientes da formação do ácido carbônico. Íons $\mathrm{H}^{+}$são preferencialmente adsorvidos pela superfície dos minerais de carga negativa presentes no meio (como caulinita e ilita), proporcionando a dessorção de outros cátions por mecanismos de troca iônica (MESTRINHO, 2008). $\mathrm{O}$ discreto aumento das concentrações de $\mathrm{Mg}$ e $\mathrm{Al}$ (0,2 mg. $\mathrm{L}^{-1}$, em ambos) pode ser explicado pela limitada dissolução mineral da caulinita (Eq. 4) e da ilita (Eq. 5) em ambiente ácido (SVERDRUP; WARFVINGE, 1988), além da pequena fração destes argilominerais presente na composição do aquífero $(<2,5 \%)$. Ao contrário, em aquíferos com altos teores de calcita e dolomita (minerais reativos e com elevada taxa de dissolução em meio ácido), a dissolução de $\mathrm{CO}_{2}$ elevou as concentrações de $\mathrm{Mg}$ e Ca em 2,8 e 2,7 vezes, respectivamente, em comparação com o background (KHARAKA et al., 2010). Peter et al. (2012) também observaram incremento entre $24 \%$ e $38 \%$ nas concentrações de $\mathrm{Ca}, \mathrm{Mg}$ e $\mathrm{K}$ em aquífero com alto teor de minerais reativos. Embora a magnitude das alterações nas concentrações iônicas observadas neste trabalho tenham sido características de aquíferos de reatividade limitada, a tendência de aumento não pôde ser estatisticamente avaliada devido à pequena quantidade de pontos amostrais (uma coleta no período de background e outra no pós-injeção).

As baixas concentrações de íons dissolvidos, características de aquíferos com reatividade limitada, resultam em pequenas variações da condutividade elétrica (CE) do meio. Os valores de $\mathrm{CE}$ sofreram alteração significativa $(\mathrm{p}<0,05)$ somente no PM 5, a 2 metros de profundidade, local em que os valores chegaram a $63 \mu \mathrm{S} . \mathrm{cm}^{-1}$, correspondendo a um aumento de $24 \%$ em relação ao valor máximo de background $\left(51 \mu \mathrm{S} . \mathrm{cm}^{-1}\right)$ (Fig. 7). Esse aumento foi observado somente no período de pós-injeção e apresentou um decréscimo ao final do monitoramento. Nos demais pontos de amostragem, os valores permaneceram constantes, abaixo de $45 \mu \mathrm{S} . \mathrm{cm}^{-1}$. Em aquíferos semelhantes, compostos predominantemente por quartzo $(>90 \%)$, reações de dissolução de pequenas quantidades de K-feldspato $(<5 \%)$ e plagioclásio $(<3 \%)$ causaram aumento da CE para $310 \mu \mathrm{S} . \mathrm{cm}^{-1}$, 90\% maior em relação ao background (CAHILL; JAKOBSEN, 2013). Em aquífero com presença de calcita, a CE chegou a 1.800 $\mu \mathrm{S} . \mathrm{cm}^{-1}$ devido à injeção de $\mathrm{CO}_{2}$, incremento 3 vezes superior ao background (KHARAKA et al., 2010). Alterações da CE em aquíferos de reatividade limitada são pontuais e, dessa forma, podem não indicar a presença de vazamentos de $\mathrm{CO}_{2}$.

Tabela 3 - Concentração máxima de íons dissolvidos observada no background e 15 dias após o término da injeção de $\mathrm{CO}_{2}$

\begin{tabular}{|c|c|c|c|c|c|c|c|c|c|c|c|c|}
\hline & $\mathrm{Ca}$ & $\mathrm{Mg}$ & $\mathrm{Na}$ & $\mathrm{K}$ & $\mathrm{S}$ & $\mathrm{P}$ & I & & $\mathrm{Al}$ & $\mathrm{Sb}$ & As & $\mathrm{Ba}$ \\
\hline $\begin{array}{l}\text { Background } \\
\text { Pós-injeção }\end{array}$ & $\begin{array}{c}<\mathrm{L} . \mathrm{Q} \cdot{ }^{1} \\
0,42\end{array}$ & $\begin{array}{l}0,10 \\
0,30\end{array}$ & $\begin{array}{l}0,97 \\
5,26\end{array}$ & $\begin{array}{l}\text { 9,90 } \\
<\text { L.Q. }\end{array}$ & $\begin{array}{l}0,31 \\
0,52\end{array}$ & $\begin{array}{r}\text {---mg.L- } \\
\text { <L.Q. } \\
\quad 0,01\end{array}$ & $\begin{array}{r}1--- \\
1,\end{array}$ & & $\begin{array}{l}0,06 \\
0,26\end{array}$ & $\begin{array}{l}0,75 \\
0,46\end{array}$ & $\begin{array}{c}<\mathrm{L} . \mathrm{Q} . \\
0,07\end{array}$ & $\begin{array}{c}<\mathrm{L} . \mathrm{Q} . \\
0,03\end{array}$ \\
\hline & $\mathrm{Be}$ & $\mathrm{Cd}$ & $\mathrm{Pb}$ & $\mathrm{Cu}$ & $\mathrm{Cr}$ & $\mathrm{Fe}$ & $\mathrm{Mn}$ & $\mathrm{Ni}$ & $\mathrm{Ag}$ & $\mathrm{Se}$ & $\mathrm{V}$ & $\mathrm{Zn}$ \\
\hline $\begin{array}{l}\text { Background } \\
\text { Pós-injeção }\end{array}$ & $\begin{array}{c}1,23 \\
<\text { L.Q. }\end{array}$ & $\begin{array}{c}<\mathrm{L} . \mathrm{Q} . \\
0,05\end{array}$ & $\begin{array}{c}<\mathrm{L} . \mathrm{Q} . \\
0,17\end{array}$ & $\begin{array}{c}<\mathrm{L} . \mathrm{Q} . \\
0,08\end{array}$ & $\begin{array}{c}<\mathrm{L} . \mathrm{Q} . \\
1,24\end{array}$ & $\begin{array}{c}0,31 \\
0,28\end{array}$ & $\begin{array}{c}{ }^{1----} \\
<\mathrm{L} . \mathrm{Q} . \\
0,06\end{array}$ & $\begin{array}{c}<\mathrm{L} . \mathrm{Q} . \\
0,02\end{array}$ & $\begin{array}{l}0,44 \\
4,52\end{array}$ & $\begin{array}{l}1,88 \\
0,41\end{array}$ & $\begin{array}{c}<\mathrm{L} . \mathrm{Q} . \\
0,12\end{array}$ & $\begin{array}{c}<\mathrm{L} . \mathrm{Q} . \\
0,40\end{array}$ \\
\hline
\end{tabular}

${ }^{1} \mathrm{~L} . \mathrm{Q} .=$ Limite de quantificação $=0,005 \mathrm{mg} \cdot \mathrm{L}^{-1}$ 


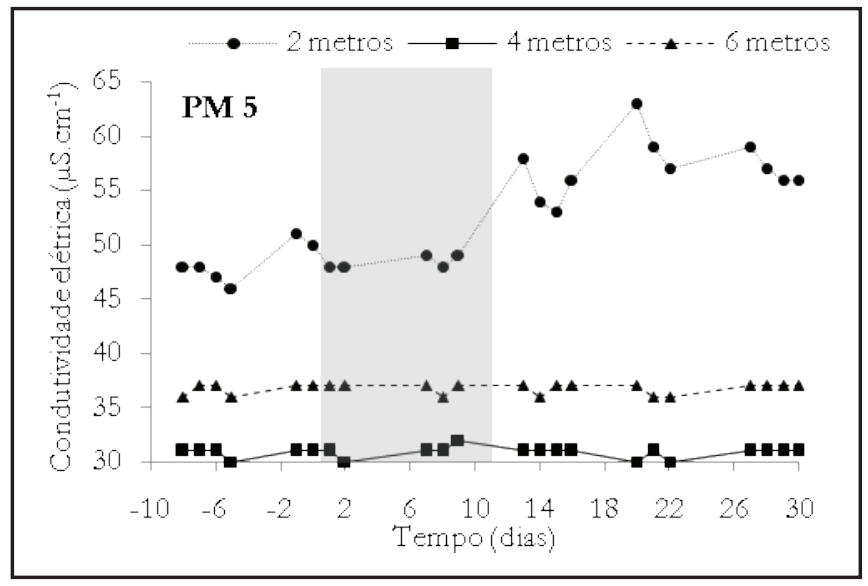

Figura 7 - Variação da condutividade elétrica no PM 5 durante o experimento. A área sombreada destaca o período de injeção de $\mathrm{CO}_{2}$ na água subterrânea

\section{CONCLUSÕES}

As interações entre $\mathrm{CO}_{2}$, água e solo determinaram a magnitude das alterações hidrogeoquímicas da água subterrânea. $\mathrm{O}$ aumento da $\not \mathrm{CO}_{2}$ causou decréscimo do $\mathrm{pH}$ para valores próximos a 4 . Este declínio foi rápido (observado a partir do segundo dia de injeção) e condicionado pela ausência de capacidade de tamponamento do aquífero, pelo predomínio de minerais de reatividade limitada $(>95 \%$ de quartzo e $<2,5$ $\%$ de argilominerais) em sua composição. A amplitude de variação do $\mathrm{pH}$ foi estatisticamente representativa dos efeitos da dissolução de $\mathrm{CO}_{2}$, considerando-se a reatividade do aquífero estudado.Assim, em aquíferos de reatividade limitada, variações de $\mathrm{pH}$ podem ser indicadoras de vazamentos de $\mathrm{CO}_{2}$ provenientes da formação geológica de armazenamento. Alterações das variáveis relacionadas à dissolução mineral (alcalinidade, condutividade elétrica e concentração de íons) e ao fluxo de $\mathrm{CO}_{2}$ gasoso (potencial de oxidação-redução) foram observadas de forma pontual, não apresentaram significância estatística e não contribuíram para a indicação de vazamentos de $\mathrm{CO}_{2}$ no aquífero. Em aquíferos de reatividade limitada, a determinação das condições de background, por meio da caracterização físicoquímica do aquífero, é de extrema importância para se avaliar com maior segurança as alterações hidrogeoquímicas da água subterrânea pela presença de $\mathrm{CO}_{2}$. Além disso, as análises hidrogeoquímicas são de fácil execução e de baixo custo, uma vez que as variáveis analisadas são de rotina dos laboratórios para determinação da qualidade de águas.

\section{AGRADECIMENTOS}

Este estudo foi financiado pelo Centro de Pesquisas Leopoldo Américo Miguez de Mello (CENPES) da Petrobras $\mathrm{S} / \mathrm{A}$ e faz parte do projeto de pesquisa intitulado "Laboratório de Campo e Desenvolvimento de Tecnologias de Medição, Monitoramento e Verificação (MMV) de $\mathrm{CO}_{2}$ para a Aplicação em Sítios de Armazenamento Geológico”. Este projeto conta com a participação de diversos núcleos de pesquisas, dentre eles, REMA (Núcleo Ressacada de Pesquisa em Meio Ambiente) - UFSC, CEPAC (Centro de Excelência em Pesquisa e Inovação sobre Petróleo, Recursos Minerais e Armazenamento de Carbono) PUCRS, LEBAC (Laboratório de Estudos de Bacias) - UNESP e IPEN (Instituto de Pesquisas Energéticas e Nucleares).

O financiamento da bolsa de mestrado foi concedido pela Coordenação de Aperfeiçoamento de Pessoal de Nível Superior (CAPES) e pelo Conselho Nacional de Desenvolvimento Científico e Tecnológico (CNPq).

\section{REFERÊNCIAS}

ASSOCIAÇÃO BRASILEIRA DE NORMAS TÉCNICAS. NBR 7181: Solo - Análise granulométrica. Rio de Janeiro, 1984.

ALTEVOGT, A. S.; JAFFE, P. R. Modeling the effects of gas phase $\mathrm{CO}_{2}$ intrusion on the biogeochemistry of variably saturated soils. Water Resources Research, v. 41, n. 9, p. 817-825, 2005.

AMERICAN PUBLIC HEALT ASSOCIATION (APHA). Standard Methods for the Examination of Wather and Wastewater: Method 3120B. Washington, DC. 18th Ed. 1992a.

AMERICAN PUBLIC HEALT ASSOCIATION (APHA). Standard Methods for the Examination of Water and Wastewater: Method 2310B. Washington, DC. 18th Ed. 1992b.

AMERICAN PUBLIC HEALT ASSOCIATION (APHA). Standard Methods for the Examination of Water and Wastewater: Method 2320B. Washington, DC. 18th Ed. 1992c.

AMERICAN PUBLIC HEALT ASSOCIATION (APHA). Standard Methods for the Examination of Water and Wastewater: Method 8146. Washington, DC. 18th Ed. 1992d.

APPS, J. A. et al. Evaluation of Potential Changes in Groundwater Quality in Response to $\mathrm{CO}_{2}$ Leakage from Deep Geologic Storage. Transport in Porous Media, v. 82, n. 1, p. 215-246, 2010.

BACHU, S.; ADAMS, J. J. Sequestration of $\mathrm{CO}_{2}$ in geological media in response to climate change: capacity of deep saline aquifers to sequester $\mathrm{CO}_{2}$ in solution. Energy Conversion and Management, v. 44, n. 20, p. 3151-3175, 2003.

BUTLER, J. N. Carbon Dioxide Equilibria and Their Applications. Addison-Wesley Publishing Company. 1982. p. 259.

CAHILL, A. G. et al. Risks attributable to water quality changes in shallow potable aquifers from geological carbon sequestration leakage into sediments of variable carbonate content. International Journal of Greenhouse Gas Control, v. 19, p. 117-125, 2013.

CAHILL, A. G.; JAKOBSEN, R. Hydro-geochemical impact of $\mathrm{CO}_{2}$ leakage from geological storage on shallow potable aquifers: A field scale pilot experiment. International Journal of 
Greenhouse Gas Control, v. 19, p. 678-688, 2013.

CARROLL, S.; HAO, Y.; AINES, R. Geochemical detection of carbon dioxide in dilute aquifers. Geochemical transactions, v. 10, p. 4, 2009.

FAHRNER, S.; SCHÄFER, D.; DAHMKE, A. A monitoring strategy to detect $\mathrm{CO}_{2}$ intrusion in deeper freshwater aquifers. International Journal of Greenhouse Gas Control, v. 9, p. 262-271, 2012.

FET'TER, C. W. Applied Hydrogeology. 3rd. ed. Upper Saddle River, New Jersey: Prentice Hall, 1994. p. 691.

GASDA, S. E.; BACHU, S.; CELIA, M. A. Spatial characterization of the location of potentially leaky wells penetrating a deep saline aquifer in a mature sedimentary basin. Environmental Geology, v. 46, n. 6-7, p. 707-720, 2004.

HARVEY, O. R. et al. Geochemical implications of gas leakage associated with geologic $\mathrm{CO}_{2}$ storage - A qualitative review. Environmental science \& technology, v. 47, n. 1, p. 23-36, 2013.

IPCC - Intergovernmental Panel on Climate Change. Special Report on Carbon Dioxide Capture and Storage. Prepared byWorking Group III of the Intergovernmental Panel on Climate Change [Metz, B.,O. Davidson, H. C. de Coninck, M. Loos, and L. A. Meyer (eds.)]. Cambridge University Press, Cambridge, United Kingdom and NewYork, NY, USA, 2005. 442 p.

IPUF - INSTITUTO DE PLANEJAMENTO URBANO DE FLORIANÓPOLIS/SC. Atlas do Município de Florianópolis. Florianópolis: PUF, 2004.

JONES, D. G. et al. New and established techniques for surface gas monitoring at onshore $\mathrm{CO}_{2}$ storage sites. Energy Procedia, v. 1, n. 1, p. 2127-2134, 2009.

JONES, D. G. et al. In Salah gas $\mathrm{CO}_{2}$ storage JIP: Surface gas and biological monitoring. Energy Procedia, v. 4, p. 3566-3573, 2011.

KHARAKA, Y. K. et al. Changes in the chemistry of shallow groundwater related to the 2008 injection of $\mathrm{CO}_{2}$ at the ZERT field site, Bozeman, Montana. Environmental Earth Sciences, v. 60, n. 2, p. 273-284, 2010.

KLUSMAN, R. W. Comparison of surface and near-surface geochemical methods for detection of gas microseepage from carbon dioxide sequestration. International Journal of Greenhouse Gas Control, v. 5, n. 6, p. 1369-1392, 2011.

KORRE, A. et al. Quantification techniques for potential $\mathrm{CO}_{2}$ leakage from geological storage sites. Energy Procedia, v. 4, p. 3413-3420, 2011.

LEBAC (LABORATÓRIO DE ESTUDOS DE BACIAS). Mapeamento geológico e hidrogeológico na área experimental de MMV de $\mathrm{CO}_{2}$ na Fazenda Ressacada. Relatório Interno
Petrobras S/A. Florianópolis. 2013.

LEUNING, R. et al. Atmospheric monitoring and verification technologies for $\mathrm{CO}_{2}$ geosequestration. International Journal of Greenhouse Gas Control, v. 2, n. 3, p. 401-414, 2008.

LEWICKI, J. L.; HILLEY, G. E. Eddy covariance network design for mapping and qualification of surface $\mathrm{CO}_{2}$ leakage fluxes. International Journal of Greenhouse Gas Control, v. 7, p. 137-144, 2012.

$\mathrm{LU}$, J. et al. Potential risks to freshwater resources as a result of leakage from $\mathrm{CO}_{2}$ geological storage: a batch-reaction experiment. Environmental Earth Sciences, v. 60, n. 2, p. 335-348, 2010.

$\mathrm{LU}$, J. et al. $\mathrm{CO}_{2}$-rock-brine interactions in Lower Tuscaloosa Formation at Cranfield $\mathrm{CO}_{2}$ sequestration site, Mississippi, U.S.A. Chemical Geology, v. 291, p. 269-277, jan. 2012.

MALE, E. J. et al. Using hyperspectral plant signatures for $\mathrm{CO}_{2}$ leak detection during the 2008 ZERT $\mathrm{CO}_{2}$ sequestration field experiment in Bozeman, Montana. Environ. Earth Sci., v. 60, n. 2, p. 251-261, 2010.

MESTRINHO, S. S. P. Geoquímica das águas subterrâneas. In: FEITOSA, F. A. C. et al. (Eds.). Hidrogeologia: Conceitos e Aplicações. 3a. ed. Rio de Janeiro: CPRM: LABHID, 2008. p. 359-379.

NOBLE, R. R. P. et al. Biological monitoring for carbon capture and storage - A review and potential future developments. International Journal of Greenhouse Gas Control, v. 10, p. 520-535, 2012.

NORDBOTTEN, J. M. et al. Model for $\mathrm{CO}_{2}$ leakage including multiple geological layers and multiple leaky wells. Environmental science \& technology, v. 43, n. 3, p. 743-9, 2009.

PAYNE, F. C.; QUINNAN, J. A.; POTTER, S. T. Remediation Hydraulics. Florida: CRC Press, 2008. p. 432.

PETER, A. et al. Investigation of the geochemical impact of $\mathrm{CO}_{2}$ on shallow groundwater: design and implementation of a $\mathrm{CO}_{2}$ injection test in Northeast Germany. Environmental Earth Sciences, v. 67, n. 2, p. 335-349, 2012.

RAMOS, D. et al. Biostimulation of anaerobic BTEX biodegradation under fermentative methanogenic conditions at source-zone groundwater contaminated with a biodiesel blend (B20). Biodegradation, v. 24, n. 3, p. 333-341, 2013.

ROUSE, J. H. et al. Multi-Spectral imaging of vegetation for detecting $\mathrm{CO}_{2}$ leaking from underground. Environmental Earth Sciences, v. 60, n. 2, 2010.

STUMM, W.; MORGAN, J. J. Aquatic Chemistry. 2nd. ed. Ney York: John Wiley \& Sons, 1981. p. 780. 
SVERDRUP, H.; WARFVINGE, P. Weathering of primary silicate minerals in the natural soil environment in relation to a chemical weathering model. Water, Air, and Soil Pollution, v. 38, p. 387-408, 1988.

TEDESCO, M. J.; GIANELlO, C.; BISSANI, C. A. Análise de solo, plantas e outros materiais. Porto Alegre: Universidade Federal do Rio Grande do Sul, 1995. p. 174.

TRAUTZ, R. C. et al. Effect of dissolved $\mathrm{CO}_{2}$ on a shallow groundwater system: a controlled release field experiment. Environmental science \& technology, v. 47, n. 1, p. 298-305, 2013.

USEPA - UNITED STATES ENVIRONMENTAL PROTECTION AGENCY. Determination of Inorganic Anions by Ion Chromatography: Method 300.00, revision 2.1.Cincinnati, Ohio, 1993.

VARADHARAJAN, C. et al. A laboratory study of the initial effects of dissolved carbon dioxide $\left(\mathrm{CO}_{2}\right)$ on metal release from shallow sediments. International Journal of Greenhouse Gas Control, v. 19, p. 183-211, 2013.

WANG, S.; JAFFE, P. R. Dissolution of a mineral phase in potable aquifers due to $\mathrm{CO}_{2}$ releases from deep formations; effect of dissolution kinetics. Energy Conversion and Management, v. 45, n. 18-19, p. 2833-2848, 2004.

WHITE, C. M. et al. Separation and capture of $\mathrm{CO}_{2}$ from large stationary sources and sequestration in geological formations - coalbeds and deep saline aquifers. Journal of the Air \& Waste Management Association, v. 53, n. 6, p. 645-715, 2003.

WHITE, D. J. Geophysical monitoring of the Weyburn $\mathrm{CO}_{2}$ flood: Results during 10 years of injection. Energy Procedia, v. 4, p. 3628-3635, 2011.

WILKIN, R. T.; DIGIULIO, D. C. Geochemical impacts to groundwater from geologic carbon sequestration: controls on $\mathrm{pH}$ and inorganic carbon concentrations from reaction path and kinetic modeling. Environmental science \& technology, v. 44, n. 12, p. 4821-7, 2010. 